\title{
Metabolic syndrome in psychiatry: advances in understanding and management
}

\author{
Cyrus S. H. Ho, Melvyn W. B. Zhang, Anselm Mak \& Roger C. M. Ho
}

\begin{abstract}
SUMMARY
Metabolic syndrome comprises a number of cardiovascular risk factors that increase morbidity and mortality. The increase in incidence of the syndrome among psychiatric patients has been unanimously demonstrated in recent studies and it has become one of the greatest challenges in psychiatric practice. Besides the use of psychotropic drugs, factors such as genetic polymorphisms, inflammation, endocrinopathies and unhealthy lifestyle contribute to the association between metabolic syndrome and a number of psychiatric disorders. In this article, we review the current diagnostic criteria for metabolic syndrome and propose clinically useful guidelines for psychiatrists to identify and monitor patients who may have the syndrome. We also outline the relationship between metabolic syndrome and individual psychiatric disorders, and discuss advances in pharmacological treatment for the syndrome, such as metformin.
\end{abstract}

\section{LEARNING OBJECTIVES}

- Be familiar with the definition of metabolic syndrome and its parameters of measurement.

- Appreciate how individual psychiatric disorders contribute to metabolic syndrome and vice versa.

- Develop a framework for the prevention, screening and management of metabolic syndrome in psychiatric patients.

\section{DECLARATION OF INTEREST}

None.

Metabolic syndrome is a major public health problem that has been recognised to be a global epidemic by the World Health Organization (WHO) (Potenza 2009). It is a constellation of clinical signs and suboptimal laboratory findings involving five key features: central obesity, elevated blood pressure, hypertriglyceridaemia, low serum levels of high-density lipoprotein (HDL) cholesterol and high serum levels of fasting glucose. It is generally agreed that the presence of at least three of these components is required before a patient can be classified as having metabolic syndrome (Grundy 2004). However, the most widely used definitions of the syndrome focus predominantly on either waist circumference (a surrogate measure of central obesity) or insulin resistance (Table 1) - criteria that apply differently to various ethnic groups.

Given the difficulty in reaching consensus among these definitions, a number of major international bodies came together in an attempt to harmonise diagnostic criteria (Alberti 2009; Kassi 2011). They advised that there should be no obligatory component for metabolic syndrome, but rather that all five individual components should be deemed important in risk prediction. These components are independent risk factors for coronary heart disease, cerebrovascular disease and diabetes.

\section{Prevalence}

Metabolic syndrome affects around $20-25 \%$ of the population worldwide, and it is associated with a twofold increase in the likelihood of death and threefold increase in the risk of heart attack or stroke (International Diabetes Federation 2006). Metabolic syndrome also has a huge impact on the financial, emotional and psychosocial well-being of those who have the disease and their families. The syndrome is associated with an increased risk of developing psychiatric disorders such as depression, resulting in reduced quality of life. Conversely, long-term psychiatric illnesses can promote metabolic syndrome: its prevalence is 2-3 times higher in people with severe psychiatric illnesses (Holt 2010).

\section{Aetiology}

The cause of metabolic syndrome in psychiatric patients is likely to be multifactorial (Box 1) (Grundy 2004). Psychotropic drugs such as secondgeneration antipsychotics and mood stabilisers are established risk factors. Negative health behaviours such as smoking, excessive alcohol consumption, lack of physical exercise, unhealthy eating habits are also likely to contribute, as are

\section{ARTICLE}

Cyrus Ho is a specialist registrar in charge of the schizophrenia, depressive disorder and anxiety disorder clinics at the National University Hospital, Singapore. He has a special interest in the interface of psychiatry and medicine. Melvyn Zhang is currently a core trainee in psychiatry with the National Healthcare Group, Singapore. Anselm Mak is an assistant professor and consultant rheumatologist at the National University Hospital, Singapore. He has special interest in internal medicine, systemic lupus erythematosus and clinical immunology. Roger Ho is an assistant professor and a consultant psychiatrist at the National University Hospital, Singapore. He has a special interest in psychoneuroimmunology and the interface between medicine and psychiatry.

Correspondence Dr Roger C.M Ho, Department of Psychological Medicine, Level 9, NUHS Tower Block, 1E Lower Kent Ridge Road, Singapore 119 228. Email: pcmrhcm@nus.edu.sg 


\begin{tabular}{|c|c|c|c|c|}
\hline Criteria & WHO & IDF & EGIR & NCEP-ATP III \\
\hline & $\begin{array}{l}\text { Diabetes plus at least two of } \\
\text { the following: }\end{array}$ & $\begin{array}{l}\text { Central obesity plus at least } \\
\text { two of the following: }\end{array}$ & $\begin{array}{l}\text { Insulin resistance plus at } \\
\text { least two of the following: }\end{array}$ & $\begin{array}{l}\text { At least three of the } \\
\text { following: }\end{array}$ \\
\hline $\begin{array}{l}\text { Central obesity/waist } \\
\text { circumference }\end{array}$ & $\begin{array}{l}\text { Waist } / \text { hip ratio }>0.90 \\
\text { (men), }>0.85 \text { (women); } \\
\text { or } \mathrm{BMl}>30 \mathrm{~kg} / \mathrm{m}^{2}\end{array}$ & $\begin{array}{l}\text { Waist circumference, based } \\
\text { on ethnicity-specific values. } \\
\text { If } \mathrm{BMI}>30 \mathrm{~kg} / \mathrm{m}^{2} \text {, waist } \\
\text { circumference does not need } \\
\text { to be measured }\end{array}$ & $\begin{array}{l}\text { Waist circumference: } \\
\geq 94 \mathrm{~cm} \text { (men), } \\
\geq 80 \mathrm{~cm} \text { (women) }\end{array}$ & $\begin{array}{l}\text { Waist circumference: }>102 \mathrm{~cm} \\
\text { (men), }>88 \mathrm{~cm} \text { (women) }\end{array}$ \\
\hline Triglycerides & $\geq 1.7 \mathrm{mmol} / \mathrm{l}$ & $\begin{array}{l}>1.7 \mathrm{mmol} / \mathrm{l} \text {, or specific } \\
\text { treatment for lipid } \\
\text { abnormality }\end{array}$ & $\begin{array}{l}\geq 2.0 \mathrm{mmol} / \mathrm{l} \text {, or treatment for } \\
\text { lipid abnormality }\end{array}$ & $>1.7 \mathrm{mmol} / \mathrm{l}$ \\
\hline $\begin{array}{l}\text { High-density lipoprotein } \\
\text { (HDL) cholesterol }\end{array}$ & $\begin{array}{l}<0.9 \mathrm{mmol} / / \text { (men), } \\
<1.0 \mathrm{mmol} / / \text { (women) }\end{array}$ & $\begin{array}{l}<1.04 \mathrm{mmol} / \mathrm{I}(\mathrm{men}), \\
<1.29 \mathrm{mmol} / \mathrm{l} \text { (women); or } \\
\text { specific treatment for lipid } \\
\text { abnormality }\end{array}$ & $<1.0 \mathrm{mmol} / \mathrm{l}$ & $\begin{array}{l}<1.04 \mathrm{mmol} / / \text { (men), } \\
<1.29 \mathrm{mmol} / / \text { (women) }\end{array}$ \\
\hline $\begin{array}{l}\text { Blood pressure (systolic/ } \\
\text { diastolic) }\end{array}$ & $\geq 140 / 90 \mathrm{mmHg}$ & $\begin{array}{l}\text { Systolic blood pressure } \\
>130 \text { or diastolic blood } \\
\text { pressure }>85 \mathrm{mmHg} \text {, or } \\
\text { antihypertensive treatment }\end{array}$ & $\begin{array}{l}\geq 140 / 90 \mathrm{mmHg} \text {, or } \\
\text { antihypertensive medication }\end{array}$ & $>130 / 85 \mathrm{mmHg}$ \\
\hline Fasting plasma glucose & Impaired & $\begin{array}{l}>5.6 \mathrm{mmol} / \mathrm{l}, \text { or previously } \\
\text { diagnosed type } 2 \text { diabetes }\end{array}$ & $\geq 6.1 \mathrm{mmol} / \mathrm{l}$ & $>6.1 \mathrm{mmol} / \mathrm{l}$ \\
\hline Urinary albumin & $\begin{array}{l}\text { Excretion rate } \geq 20 \mu \mathrm{g} / \mathrm{min} \text {, } \\
\text { or albumin } / \text { creatinine ratio } \\
\geq 30 \mathrm{mg} / \mathrm{g}\end{array}$ & Not included & Not included & Not included \\
\hline
\end{tabular}

WHO, World Health Organization (1999); IDF, International Diabetes Federation (2006); EGIR, European Group for the Study of Insulin Resistance (Balkau 1999); NCEP-ATP III, US National Cholesterol Education Program Adult Treatment Panel III (Expert Panel on Detection, Evaluation, and Treatment of High Blood Cholesterol in Adults 2001); BMI, body mass index. Source: Bloomgarden (2004).

biological factors such as hypothalamic-pituitaryadrenal (HPA) axis dysregulation and insulin resistance. Inflammation is recognised as an integral component of both metabolic syndrome and psychiatric disorders (Hope 2009; Na 2012), with high serum levels of C-reactive protein (CRP) and proinflammatory cytokines such as interleukin IL-6 and tumour necrosis factoralpha (TNF- $\alpha$ ) in both conditions. Genetic factors such as the presence of specific genes responsible for a predisposition to metabolic problems and variations in the leptin-melanocortin pathway that contribute to heterogeneity in antipsychoticinduced weight gain are also important causes of metabolic syndrome.

B0X 1 Common risk factors for metabolic syndrome in chronic psychiatric illness

- Excessive alcohol consumption

- Food imbalance and poor dietary habits

- Genetic predisposition

- Hormonal imbalances involving cortisol and leptin

- Second-generation antipsychotics and their related side-effects

- Sedentary lifestyle
The relationship between metabolic syndrome and psychiatric illness is indeed intricate and likely confounded by many issues. Nevertheless, the aim of this article is to highlight some of the mechanisms underlying the two and to outline potential treatment strategies.

\section{Schizophrenia and metabolic syndrome}

\section{Epidemiology}

Metabolic syndrome is common among people with schizophrenia, although the estimated prevalence varies widely, from 8.9 to $68 \%$. This wide range may be attributed to the different age groups and ethnic groups studied and the use of different diagnostic criteria (McEvoy 2005). Nevertheless, the prevalence of metabolic syndrome in people with schizophrenia is still around 5 times higher than that in the general population. Women with schizophrenia have a higher risk of developing metabolic syndrome compared with men (McEvoy 2005). Mortality among people with schizophrenia is two- to threefold higher than in the general population, and this is largely accounted for by cardiovascular morbidity (Koponen 2010).

Schizophrenia is also associated with a greater risk of diabetes mellitus, with a two- to threefold higher prevalence compared with the general population (de Hert 2009). This increase is 
independent of the use of antipsychotic drugs. Antipsychotic-naive patients were found to have higher insulin resistance, impaired glucose tolerance and increased intra-abdominal fat deposition compared with normal controls (Papanastasiou 2012). In addition, siblings of individuals with schizophrenia were found to have increased glucose intolerance (Fernandez-Egea 2008a), while parents of those with non-affective psychosis had increased prevalence of type 2 diabetes (Fernandez-Egea 2008b). These findings suggest that metabolic abnormality is probably an intrinsic component of schizophrenia, with biological and genetic predisposing factors.

\section{Common genetic factors}

Research suggests a possible common genetic predisposition to both metabolic syndrome and schizophrenia. An at-risk allele of type 2 diabetes, rs7903146[T], has been found in the transcription factor 7-like 2 (TCF7L2) gene and this genotype is also associated with an increased risk of schizophrenia (Hansen 2011). Associations between weight gain in patients with schizophrenia and various genetic polymorphisms have also been identified. For example, the alpha$1 \mathrm{~A}$ adrenergic receptor $(A D R A 1 A)$ gene is related to cardiovascular risk factors such as obesity and hypertension, and a positive association has been reported between the cumulative number of metabolic syndrome components and the presence of the Arg347 allele of ADRA1A (Cheng 2012).

\section{Chronic inflammation}

Many studies have reported chronic subclinical inflammation to be part of metabolic syndrome (Newcomer 2007). Although inflammatory markers are not currently included in the diagnostic criteria for metabolic syndrome, they have been linked to it, particularly CRP, TNF- $\alpha$, IL-6, adiponectin and leptin, which are present in visceral fat (Sutherland 2004). Adipose tissue of obese people secretes higher amounts of these inflammatory molecules compared with adipose tissue of lean individuals. In addition, infiltration of macrophages and inflammationrelated gene expression in adipose tissue may precede the development of insulin resistance. Insulin resistance in turn has been postulated to promote pro-inflammatory cytokine production (McLaughlin 2002). Adipose-derived hormones such as adiponectin and leptin are important in controlling energy homeostasis and glucose and lipid metabolism. Adiponectin, which is lowered with rising adiposity, is positively associated with insulin sensitivity and has anti-atherogenic properties that prevent the development of atherosclerotic plaques via inhibition of TNF- $\alpha$ production by macrophages. As for leptin, which may impair insulin action, it enhances inflammation by increasing the synthesis of TNF- $\alpha$ and IL-6. There is growing evidence to suggest that inflammation contributes to the development of metabolic syndrome in patients with schizophrenia (Na 2012). Both schizophrenia and obesity demonstrate a decrease in adiponectin and increase in leptin, with raised TNF- $\alpha$ and IL-6. This leads to inflammation, which ultimately confers higher risks of atherosclerosis and coronary artery disease.

Blood serum levels of CRP, TNF- $\alpha$ and homocysteine have been found to be increased in patients with schizophrenia (Hope 2009). C-reactive protein was found to have a positive association with waist circumference and diastolic blood pressure, while homocysteine has a positive association with waist circumference, systolic and diastolic blood pressure, triglycerides and glucose (Vuksan-Cusa 2012). Abnormal baseline levels of IL-6 were found to predict significantly greater elevations in both total cholesterol and low-density lipoprotein (LDL) following a 12-week treatment with olanzapine (Fernandez-Egea 2011). Total white blood cell count, one of the surrogate parameters of inflammation, has been found to be a risk factor for metabolic syndrome, its values positively correlating with the increase in waist circumference and glucose during a 24 -week treatment with paliperidone ( $\mathrm{Na} 2012$ ).

\section{Lifestyle factors}

People with schizophrenia often have a more sedentary lifestyle, with little physical exercise, poor dietary habits and more smoking, all of which contribute to metabolic syndrome (Connolly 2005). These are partly attributed to negative symptoms of schizophrenia, lack of motivation, poor insight into their health, and sedation associated with antipsychotics. Smoking in particular has a negative effect on treatment, as it induces hepatic enzymes, thereby increasing metabolism of psychotropic medications. Thus, higher doses of antipsychotics may be required for smokers than for non-smokers.

\section{Antipsychotics}

Antipsychotics are the mainstay of management of schizophrenia: there is compelling evidence that they reduce morbidity, suicide and hospital admissions. Compared with first-generation antipsychotics, the second-generation drugs cause fewer extrapyramidal side-effects and offer better 
B0X 2 Second-generation antipsychotics and metabolic syndrome

Ranking on the basis of relative risk for development of metabolic syndrome:

1 Clozapine (highest risk)

2 Olanzapine

3 Quetiapine

4 Risperidone

5 Aripiprazole

6 Ziprasidone (lowest risk)

symptom control and cognitive and affective function. Their use has therefore increased. However, there is evidence that second-generation antipsychotics induce more weight gain than their predecessors, a finding especially pronounced for clozapine and olanzapine (Box 2).

\section{Antipsychotic-induced weight gain}

The prevalence of metabolic syndrome in patients with first-episode schizophrenia who have been treated with antipsychotics has been estimated at around 10\% using the NCEP-ATP III criteria and $18 \%$ using the IDF criteria (the criteria are outlined in Table 1) (Saddichha 2008). A recent study has shown an increment of 29.3 points in body mass index (BMI) percentile for children and adolescents up to 16 years of age who were on risperidone for 6 months (Goeb 2010).

The proposed mechanism of antipsychoticinduced weight gain involves multiple pathways. Selective antagonism of antipsychotics at the 5 - $\mathrm{HT}_{2 \mathrm{C}}, \mathrm{H}_{1}$ and $\mathrm{D}_{2}$ receptors is contributory. In fact, antagonism at serotonin $5-\mathrm{HT}_{2 \mathrm{C}}$ receptors increases insulin resistance and reduces glucose uptake by skeletal muscles, thereby heightening the risk of diabetes. Histamine $\mathrm{H}_{1}$ and $\mathrm{H}_{3}$ receptors are recognised as mediators of energy intake and expenditure, and histamine agonists are able to attenuate weight gain. Antipsychotics also have antihistaminergic properties, as they compete with histamine for binding sites on the $\mathrm{H}_{1}$ receptors, which leads to sedation and reduction in metabolism.

Twin studies have led to suggestions of genetic variability in antipsychotic-induced weight gain (Gebhardt 2010). Genetic variations have been proposed for the HTR2C gene, which encodes for $5-\mathrm{HT}_{2 \mathrm{C}}$ receptors. For example, a single nucleotide polymorphism (SNP) in the coding region 68G/C results in an amino acid substitution (Reynolds 2005). Polymorphisms have been found in other genes, such as the dopamine receptor $\mathrm{D}_{2}$ gene
$D R D 2$, brain-derived neurotrophic factor gene $B D N F$, and $A D R A 1 A$ (Lee 2011).

Genetic variations involving the satiety pathways have also been implicated (Lee 2011). The leptin system regulates appetite and energy metabolism via the melanocortin system (alpha-melanocytestimulating hormone and agouti-related peptide) and neuropeptide Y. Polymorphisms in the leptin gene $L E P$ and leptin receptor gene $L E P R$ are associated with increased risk of developing metabolic syndrome.

Interestingly, clozapine has been found to alter the activity of the AMP-activated protein kinaseacetyl CoA carboxylase-carnitine palmitoyl transferase 1 pathway (the AMPK-ACC-CPT1 pathway) in the rat frontal cortex. This finding suggests that clozapine influences the lipid metabolic regulatory pathway via the central nervous system (Kim 2012). However, further study is necessary to characterise the physiological relevance in human models.

There is an increasing trend of combining different antipsychotics in treating patients with treatment-resistant schizophrenia. Correll et al (2007) reported that the prescription of multiple antipsychotics was associated with a significant increase in the incidence of metabolic syndrome (50\%) compared with antipsychotic monotherapy (34\%). Conversely, the combination of clozapine and aripiprazole has been found to reduce triglyceride levels, LDL cholesterol, BMI and waist circumference, as well as negative symptoms, in patients with schizophrenia and metabolic syndrome (Fleischhacker 2010). This is probably because aripiprazole is a partial dopamine agonist that stimulates extracerebral presynaptic $\mathrm{D}_{2}$ receptors, which decreases sympathetic tone and anabolic effects, thereby reducing the risk of metabolic syndrome.

\section{Depressive disorder and metabolic syndrome}

Metabolic syndrome is associated with increased prevalence of depressive disorder and depressive symptoms, but not anxiety disorder or anxiety symptoms (Takeuchi 2009). The prevalence of metabolic syndrome in patients with depression ranges from 36 to $50 \%$. The syndrome and its components, especially waist circumference, are predictive factors for the development of depressive disorder (Takeuchi 2009). Similarly, the number of components of metabolic syndrome is correlated with higher depression score on the Hospital Anxiety and Depression Scale (Skilton 2007). Capuron et al (2008) found that depressive symptoms in patients with metabolic syndrome were predominantly neurovegetative (e.g. fatigue, anhedonia and loss of 
energy) and were less likely to be associated with affective and cognitive features.

\section{Interactive mechanisms}

The interaction between depressive disorder and metabolic syndrome is complex and is mediated by a number of factors. First, people with depression are less likely to abide by dietary restrictions and more likely to be physically inactive. They also tend to adopt unhealthy habits such as smoking and alcohol misuse. This behaviour contributes to obesity and subsequent insulin resistance (Attvall 1993; Wojciech 2007). The lack of exercise may also reduce serotonin synthesis, thus worsening depression. The psychological stress associated with the complications of metabolic syndrome may worsen depressive symptoms.

Second, the activation of the HPA axis increases plasma cortisol levels (Bjomtorp 2000). Chronic elevation of cortisol levels can lead to a pseudoCushing's syndrome, which is characterised by increased visceral adiposity, hyperinsulinaemia, insulin resistance, hypertension and dyslipidaemia, all of which are hallmarks of metabolic syndrome.

Third, the chronic increase of insulin and leptin may activate the sympathetic nervous system. This can result in elevation of the circulating catecholamine level and subsequent faulty glucose metabolism and blood pressure regulation, and accumulation of abdominal fat (Musselman 1998; Anagnostis 2009).

Fourth, the increased levels of proinflammatory cytokines (Howren 2009) and leptin resistance (Patel 2008) found in patients with metabolic syndrome have been shown to be involved in depressive disorder.

Fifth, vascular endothelial dysfunction resulting from decreased levels of vascular endothelial growth factor, which is important in neurogenesis, and the mediating effects of inflammatory cytokines affect cerebral blood flow in distinct areas of the brain, increasing the risk of depressive disorder. Bench et al (1992) found that the severity of depressive mood and psychomotor retardation was inversely correlated with regional cerebral blood flow (rCBF) in the left dorsolateral prefrontal cortex and left angular gyrus. Conversely, the severity of anxiety, insomnia and agitation were positively correlated with bilateral rCBF in the posterior cingulate cortex and inferior parietal lobule.

\section{Antidepressants}

The use of antidepressants increases the incidence of metabolic syndrome among patients with depression. Tricyclic antidepressants can cause insulin resistance and hypertriglyceridaemia, and patients taking tricyclics such as amitriptyline and doxepin have been reported to experience substantial weight gain (Chokka 2006). Although second-generation antidepressants such as selective serotonin reuptake inhibitors (SSRIs) cause an initial reduction in weight, their longterm use causes weight gain (Chokka 2006). The risk of developing metabolic syndrome is further compounded by reduction in body metabolism in patients with depression. Nevertheless, a trial of 4 -week reboxetine treatment demonstrated significant decrease in total cholesterol, LDL cholesterol, BMI and mean systolic blood pressure (Paslakis 2011). The authors suggested that these findings could possibly be attributed to the pharmacological profile of reboxetine in terms of its selective noradrenergic reuptake inhibition and lack of additional undesirable binding properties of other antidepressants (e.g. tricyclic antidepressants and $\mathrm{H}_{1}$-receptor antagonism) (Paslakis 2011). However, the study was limited by its small sample size, lack of control group and short duration of follow-up.

\section{Bipolar disorder and metabolic syndrome}

The relationship between metabolic syndrome and bipolar disorder has not been much studied, but it is increasingly recognised that patients with bipolar disorder have higher risk of the syndrome than the general population (Chi 2013). The prevalence of metabolic syndrome in bipolar disorder has been estimated to be between 25 and 27\% (Lee 2010). The aetiological factors for metabolic disturbances in people with bipolar disorder are multifactorial. Dysregulation of the HPA axis with subsequent glucocorticoid resistance, impaired glucose tolerance and insulin resistance, dysregulation of the sympathetic nervous system, increased proinflammatory cytokine production across both phases of bipolar disorder (Kim 2007) and an unhealthy lifestyle are all implicated.

Several studies have reported that metabolic syndrome tends to be associated with certain characteristics of bipolar disorder (Chang 2009; McIntyre 2010):

- longer duration of bipolar disorder, especially type I

- more lifetime manic and depressive episodes

- more severe first affective episode

- late onset of first manic episode

- late age at first treatment for manic or depressive episode.

Metabolic syndrome appears to affect the course of bipolar disorder. Individuals with bipolar disorder who have diabetes mellitus are 
more likely to experience rapid cycling, lower level of functioning and more psychiatric hospital admissions than those without diabetes mellitus (Ruzickova 2003). High rates of lifetime suicide attempts in patients with bipolar disorder and metabolic syndrome have also been reported (Fagiolini 2005).

The prevalence of metabolic syndrome in patients with type II bipolar disorder has been found to be lower than that in type I (Chi 2013). Several factors were postulated for this. For example, patients with hypomania are less likely to receive antipsychotics, and the variation in mood symptomatology may contribute to different degrees of metabolic disturbance. However, data regarding the impact of different mood states on metabolic profile are lacking.

\section{Mood stabilisers}

Mood stabilisers such as lithium and sodium valproate are first-line agents in the management of bipolar disorder, with augmentation by antipsychotics to achieve optimal mood control. Mood stabilisers, particularly lithium and sodium valproate, have been associated with metabolic syndrome. For example divalproex, a valproic acid derivative, can cause insulin resistance and weight gain. The simultaneous treatment with mood stabilisers and antipsychotics or the concurrent use of two or three mood stabilisers is associated with significantly higher metabolic disturbances (Chang 2009).

\section{Cognitive decline, dementia and metabolic syndrome}

Metabolic syndrome is associated with cognitive decline, Alzheimer's disease and vascular dementia. Elderly people with metabolic syndrome are more likely to develop cognitive impairment than those without the syndrome (Yaffe 2004). Common cognitive deficits that are linked to metabolic syndrome involve memory, visuospatial abilities, executive functioning, processing speed and overall intellectual functioning (Yates 2012). The relationship between metabolic syndrome and mild cognitive impairment (MCI) is less established, but likely to be complex. Roberts et al (2010) have proposed that it depends on the subtype of MCI and the extent of inflammation. For instance, patients with metabolic syndrome and high serum levels of CRP are more likely to develop non-amnestic MCI, not but the other subtypes.

The effects of metabolic syndrome on the brain, and thus on cognitive decline, include neuroinflammation, oxidative stress, impaired glucose metabolism and impairment of vascular reactivity (Yates 2012). In insulin resistance, there is excess production of proinflammatory cytokines such as IL-1 $\beta$, IL- 6 and TNF- $\alpha$, of which IL- $1 \beta$ and IL-6 stimulate the over-expression of amyloid$\beta$-precursor protein $(\mathrm{A} \beta \mathrm{PP})$ and deposition of amyloid- $\beta$ in the brain. In turn, amyloid- $\beta$ leads to further production of proinflammatory cytokines and worsens cognitive function in a vicious circle. Furthermore, these proinflammatory cytokines can accelerate atherosclerosis and potentially lead to irreversible structural brain changes and Alzheimer's disease (de la Torre 2010), in addition to vascular dementia. It has been increasingly recognised that vascular factors may have a role in the development of Alzheimer's disease. Impaired cerebrovascular reactivity with increased carotid stiffness and intima-media thickness has also been reported in metabolic syndrome (Koivistoinen 2009). These affect the cerebral blood flow, transportation of nutrients and clearance of metabolic waste, leading to disruptions of neuronal activities and possible progression to cognitive decline.

Interestingly, adiponectin, which is released from adipose tissue, has also been found to be related to cognitive function in several studies. Une et al (2011) discovered that plasma adiponectin was significantly higher in individuals with MCI or Alzheimer's disease than in normal controls, whereas cerebrospinal fluid adiponectin was significantly higher in those with MCI. Furthermore, the Framingham Heart Study revealed that high plasma levels of adiponectin were a risk factor for all-cause dementia and Alzheimer's disease in women (van Himbergen 2012).

These findings contradict the commonly accepted belief that a high level of plasma adiponectin is protective of metabolic and cardiovascular functioning. Nevertheless, it is important to note that the renal clearance of adiponectin in the elderly is lower than in younger adults. Thus, plasma adiponectin levels in elderly people should be interpreted with caution. In light of the above evidence, modification of diet and lifestyle as well as appropriate pharmacotherapy for hypertension, dyslipidaemia and hyperglycaemia have important implications in the prevention and treatment of Alzheimer's disease.

\section{Other psychiatric disorders and metabolic syndrome}

\section{Post-traumatic stress disorder}

Post-traumatic stress disorder (PTSD) is associated with cardiovascular risk factors such as hypertension, diabetes and obesity, and chronic and more severe PTSD may be associated with 
higher risk of metabolic syndrome (Heppner 2012). Stress-related dysregulation of glucose and lipid metabolism in PTSD can also lead to the development of metabolic syndrome.

\section{Binge eating disorder}

The reported prevalence of metabolic syndrome in obese individuals with binge eating disorder is between 50 and $60 \%$. Binge eating is associated with excess insulin secretion, impaired fasting glucose and glucose tolerance, and elevated serum lipid levels (Taylor 1999). Bulimia nervosa, which has features of binge eating, is also closely related to the severity of obesity. Furthermore, in obese patients rapid eating is associated with increased serum cholesterol and triglycerides, higher waist/ hip circumference ratio and fatty liver (Kral 2001).

\section{Borderline personality disorder}

The prevalence of metabolic syndrome among people with borderline personality disorder is twice that in primary care patients (Kahl 2013). The increased rate is associated with older age, higher BMI, second-generation antipsychotics, benzodiazepine dependency and binge eating behaviour. Hyperglycaemia is more common in both genders, with central obesity and hypertriglyceridaemia more common in women.

Several aetiologies of the associations between metabolic syndrome and borderline personality disorder have been postulated. Dysregulation of the HPA system has been demonstrated in several studies to result in hypercortisolism, imbalance of pro- and anti-inflammatory cytokines, and lowered feedback sensitivity, with or without major depressive disorder (Kahl 2006; Purnell 2009). Reasons for this phenomenon were not well elucidated, although increased cortisol in people with borderline personality disorder might result from their recurrent state of severe inner stress and tension. Unhealthy lifestyle, comorbid psychiatric conditions and use of psychotropic drugs have also been implicated in metabolic syndrome in this group.

\section{Alcohol}

Alcohol can either encourage or prevent metabolic syndrome and this is very much dependent on the amount and type of alcohol consumed (Wojciech 2007). Moderate alcohol consumption, in particular red wine, is associated with decreased incidence of metabolic syndrome, with beneficial effects on plasma lipid and glucose levels and waist circumference. This is due to the polyphenols in red wine, which increase the activity of endothelial nitric oxide synthase (eNOS). Decreased activity of eNOS can contribute to hypertension, insulin resistance and dyslipidaemia. In those who misuse alcohol, the distinct disturbances of carbohydrate and lipid metabolism can result in increased risk of hypertension, impaired fasting glucose, high triglyceridaemia and abdominal obesity.

Heavy alcohol consumption is associated with serious health problems, including malnutrition, cardiovascular disease, chronic pancreatitis, cognitive impairment and damage to almost every organ system in the body. When associated with alcoholism, it can also coexist with, contribute to or result from several different psychiatric disorders. It is of particular concern in countries where easy accessibility of alcohol and an accepting and prevalent 'drinking culture' facilitate excessive consumption.

Psychiatric disorders commonly associated with alcoholism include major depression, bipolar disorder, schizophrenia, anxiety disorders and personality disorders. It also increases risk of suicide and violence.

\section{Choosing a psychotropic medication}

The initial selection of a drug to treat a particular psychiatric disorder is important and should be based on risk-benefit profile. Changing medications later in an attempt to reverse metabolic abnormalities may lead to relapse of psychiatric symptoms. This is especially true when the patient has already experienced good clinical response to the prevailing drug regimen. Thus, it is necessary to weigh the benefits and risks of each medication before switching. For example, if the patient has pre-existing metabolic syndrome first-generation antipsychotics should be considered for schizophrenia, given that their metabolic risk profile is more favourable than that of the second-generation drugs. Saddichha et al (2008) recommend that treatment be started with a second-generation drug for a limited period (e.g. 6 weeks) and switched to a first-generation alternative when the risk of developing metabolic syndrome is high.

The data demonstrate an increased incidence of metabolic syndrome in individuals treated with multiple psychotropic drugs, so the combination of psychotropic medications should also be based on careful risk-benefit analysis.

\section{Screening for metabolic syndrome}

In focusing on the psychiatric needs of their patients, mental health professionals may not address the increased risk of metabolic syndrome, particularly in people receiving long-term antipsychotics. There is little information for 

psychiatric illnesses

\begin{tabular}{|c|c|}
\hline Time & Recommended steps \\
\hline Baseline & $\begin{array}{l}1 \text { History: include previous cardiovascular diseases, family history, smoking, } \\
\text { frequency and type of exercise, and dietary habits } \\
2 \text { Physical examination: include blood pressure, weight, waist circumference } \\
\text { and body mass index } \\
3 \text { Laboratory tests: include fasting glucose, fasting lipids, total cholesterol, } \\
\text { low-density lipoproteins (LDL), high-density lipoproteins (HDL), triglycerides } \\
\text { (TG), alanine aminotransferase (ALT) and } \gamma \text {-glutamyltransferase (GGT) } \\
4 \text { Psychoeducation: include advice on smoking cessation, careful food choices } \\
\text { and physical activity } \\
5 \text { Choice of psychotropic medication: should be based on the cardiometabolic } \\
\text { risk profile of each medication } \\
6 \text { Referral: refer to a primary care physician or specialist if there is at least one } \\
\text { abnormal finding at step } 2 \text { and/or three abnormal results at step } 3\end{array}$ \\
\hline Week 6 & $\begin{array}{l}\text { Repeat steps } 2,3 \text { and } 4 \\
\text { Monitor consumption of alcohol and cigarettes } \\
\text { Review the choice of psychotropic drugs in patients with more than } 7 \% \\
\text { increase in body weight }\end{array}$ \\
\hline Week 12 & $\begin{array}{l}\text { Repeat steps 2, } 3 \text { and } 4 \\
\text { Monitor consumption of alcohol and cigarettes }\end{array}$ \\
\hline Week 52 & $\begin{array}{l}\text { Repeat steps } 2,3 \text { and } 4 \\
\text { Monitor consumption of alcohol and cigarettes }\end{array}$ \\
\hline After 1 year & $\begin{array}{l}\text { If all the laboratory results are within normal ranges, repeat steps } 2,3 \text { and } 4 \\
\text { annually }\end{array}$ \\
\hline
\end{tabular}

Sources: de Hert, 2009; Oh, 2011.

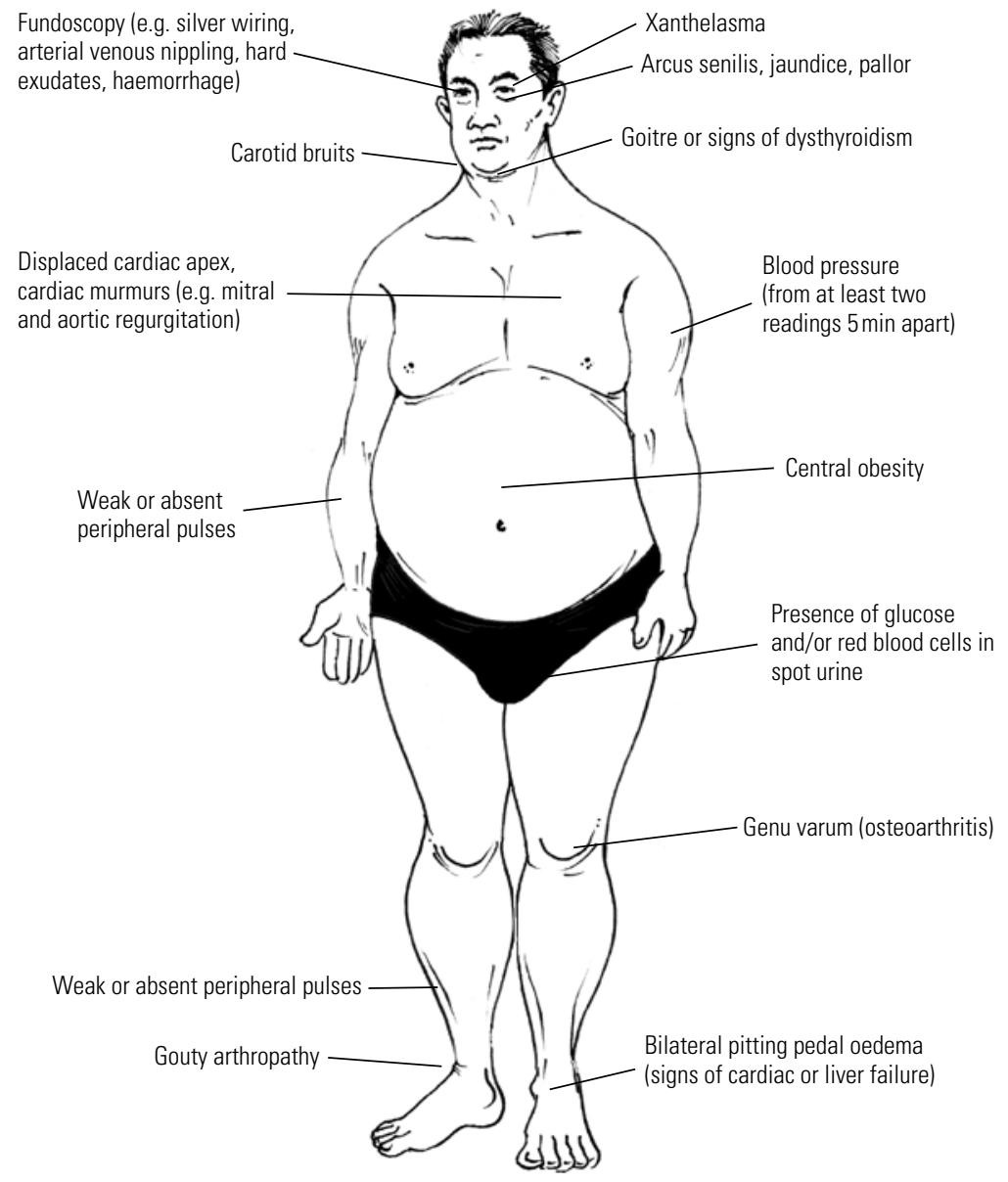

patients about lifestyle modifications to reduce the overall incidence of metabolic syndrome, and the components of the syndrome are not routinely assessed in patients with psychiatric illnesses. For example, a study conducted in southern England to assess screening of people with serious mental illnesses for metabolic abnormalities published worrying results (Holt 2010). In the preceding 12 months, the proportions of patients assessed were as follows: $32 \%$ for blood pressure; $16 \%$ for glucose; $9 \%$ for lipids; and $2 \%$ for body weight. Furthermore, fewer than a half of the in-patients and fewer than a quarter of the out-patients agreed to a physical health check. This study reflects that clinicians often underestimate the necessity to routinely screen and monitor for metabolic syndrome and that psychiatric patients are often reluctant to undergo health screening.

\section{Screening schedule and standard assessments}

Psychiatrists should screen their patients for comorbid metabolic syndrome both before and during treatment with psychotropic drugs. The suggested schedule for screening and monitoring is outlined in Table 2.

Common signs of metabolic syndrome are illustrated in Fig. 1. Weight gain, especially central obesity, is the most noticeable sign of possible metabolic syndrome. Thus, the quickest and easiest way to identify patients at risk is to measure the waist circumference.

All patients who come to the clinic for followup should routinely have their blood pressure and pulse rate measured.

Biological parameters provide an objective indication of the progress of metabolic syndrome. Besides checking fasting blood glucose level, early identification of raised LDL levels among psychiatric patients was recommended by Christoph et al (2006). For psychiatric patients with metabolic syndrome, a target LDL level less than $130 \mathrm{mg} / \mathrm{dl}$ is recommended. For those with other comorbid conditions, such as diabetes mellitus, coronary artery disease, abdominal aortic aneurysm and peripheral artery diseases, the recommended target is lower than $100 \mathrm{mg} / \mathrm{dl}$.

It is recommended that alanine aminotransferase (ALT) and $\gamma$-glutamyltransferase (GGT) are measured before initiating psychotropic drugs, as this may identify patients with potential risk of developing fatty liver disease and liver damage (Lee 2004; Park 2004). Furthermore, ALT is a predictor for mortality as a result of unrecognised liver disease, and elevated GGT levels are associated with diabetes mellitus, insulin resistance, alcoholism and cardiovascular disease. 


\section{Novel biomarkers}

Other novel potential biomarkers for metabolic syndrome, such as high-sensitivity CRP (hsCRP), homocysteine and serum uric acid, have been investigated. The role and association of CRP and homocysteine with metabolic syndrome have been explained earlier. Increased serum uric acid is associated with systemic inflammation, endothelial dysfunction, hypertension and cardiovascular diseases. Simultaneously high serum uric acid and hsCRP levels significantly associate with metabolic syndrome independent of other confounding factors. Thus, the use of these potential biomarkers as routine tests for risk stratification of metabolic syndrome in psychiatric patients should be further evaluated.

\section{Treatment of metabolic syndrome}

\section{Lifestyle modification, psychoeducation and self-help groups}

Psychiatrists need to promote healthy lifestyle practices in patients with metabolic syndrome. General recommendations for managing the syndrome are summarised in Box 3. Patients need to be educated about their psychiatric illness and medication, as this is important in enhancing medication adherence and relapse prevention.

If simple advice to eat a balanced diet and take regular exercise is insufficient, patients can be referred to dieticians and weight management specialists for more detailed dietary and exercise advice to help them lose weight. Ideally, patients should have access to holistic well-being programmes that bring these services together. Engaging patients in support groups (e.g. for weight loss) may motivate them to stay healthy. It is essential to involve family members and carers early in the treatment, to provide patients with additional support. Cognitive-behavioural therapy can be used to modify negative healthrelated beliefs and behaviours that may be detrimental to health, such as alcohol misuse and cigarette smoking.

\section{Pharmacological intervention}

The mainstay of pharmacological management of metabolic syndrome is to treat the particular component of the syndrome that is impaired. For hypertension, dyslipidaemia and diabetes, the use of antihypertensive, cholesterol-lowering and diabetic medications is indicated respectively. Such patients should be referred to primary care physicians or appropriate specialists (if there are multiple comorbidities or complications) for continued follow-up and treatment as necessary.
B0X 3 General recommendations for managing metabolic syndrome

- Diet modification - advise the patient to:

- avoid saturated fat (eg red meat, egg yolks, fried food)

- eat food low in calories

- eat fresh fruit and green vegetables

- Encourage moderate exercise (e.g. walking, swimming, cycling) for 30-40 minutes a day, three or four times a week

- Set the patient the target of losing weight between each out-patient visit

- Control blood pressure using beta-blockers to achieve $<140 / 80 \mathrm{mmHg}$

- Optimise lipid levels by diet modification or statins: aim to achieve fasting LDL cholesterol $<3 \mathrm{mmol} /$; HDL cholesterol $>1 \mathrm{mmol} / \mathrm{l}$; triglycerides $<20 \mathrm{mmol} / \mathrm{l}$

(Gurnell 2001)

It is important to be mindful of the potential interactions between these drugs and psychotropic medication, which may complicate treatment. For instance, the use of an angiotensin-converting enzyme (ACE) inhibitor to treat hypertension is contraindicated in a patient on lithium, as it can potentiate lithium toxicity. Selective serotonin reuptake inhibitors may increase risk of bleeding in a patient taking warfarin for heart treatment. In addition, some psychotropic medications can worsen control of metabolic syndrome. For example, venlafaxine can increase blood pressure and mirtazapine can increase appetite.

\section{Weight-loss drugs}

A number of studies have been performed to assess various drugs on their propensity for weight reduction. A meta-analysis of 32 randomised controlled trials that focused on pharmacological intervention for weight loss in antipsychotic-induced weight gain revealed the following order of efficacy, from most to least efficacious: metformin, D-fenfluramine, sibutramine, topiramate, reboxetine, amantadine, nizatidine, orlistat, metformin plus sibutramine, famotidine, dextroamphetamine, fluoxetine, rosiglitazone (Maayan 2010).

Metformin This biguanide, with a dual mechanism of decreasing body weight gain and improving insulin sensitivity, is most efficacious in promoting weight loss in patients with antipsychoticinduced weight gain (Mclntyre 2012). Patients with olanzapine-induced weight gain achieved a weight reduction of $5 \%$ after metformin treatment (Praharaj 2011). However, metformin seems to be effective only in reducing weight gain once it 
has occurred - it does not prevent weight gain if started concomitantly with antipsychotics (Papanastasiou 2012). Although these studies have provided encouraging preliminary evidence on the potential use of metformin in promoting weight loss and improving insulin resistance induced by antipsychotics, further large randomised, placebocontrolled studies are needed to evaluate its use.

Topiramate The anti-epileptic topiramate may be helpful in nocturnal eating syndrome and sleeprelated eating disorder (McElroy 2009). Its antibinge eating and anti-purging effects are particularly useful in patients with binge eating disorders.

Bupropion plus naltrexone Another potential treatment is the combination of bupropion and naltrexone. Combined, their mode of action is related to the modulation of hypothalamic proopiomelanocortin (POMC) neurons and the mesolimbic pathway, both of which are important in regulation of food intake and body weight (Mclntyre 2012).

Novel drugs New drugs have been used in the treatment of metabolic syndrome. Endogenous incretins including glucagon-like peptide-1 (GLP1) and glucose-dependent insulinotropic peptide (GIP) regulate insulin secretion (Nauck 2011). As GLP-1 is degraded by dipeptidyl peptidase-4 (DPP4), DPP-4 inhibitors and synthetic GLP-1 analogs form the basis of incretin-based therapies for type 2 diabetes mellitus. Glucagon-like peptide-1 (GLP1) analogues such as exenatide and liraglutide are associated with modest reductions in body weight and blood pressure in metabolic syndrome. Thus, it would be worthwhile evaluating these drugs for their safety and efficacy in the management of metabolic syndrome in psychiatric patients.

\section{Surgical intervention}

${ }^{\dagger}$ For a detailed discussion of bariatric surgery in Advances, see Stevens T et al (2012) Your patient and weight-loss surgery, 18 : 418-425. Ed. and 39 and more than one obesity-associated comorbidity, or a BMI >40 without medical judicious detection and management of metabolic disturbances in psychiatric patients are of paramount importance. It is crucial to recognise and treat metabolic syndrome, as the constellation of symptoms is associated not only with an elevated cardiovascular risk, but also with a higher prevalence of psychotic and depressive symptoms, poor perceived physical health and lower adherence to medications.

Psychiatric patients with metabolic syndrome should ideally be attended by a multidisciplinary team consisting of medical specialists, psychiatrists and dieticians, and the patients should assume an active role in their own care. The challenge lies in engaging such patients, who can be more susceptible to impulsivity, risky health behaviour and sedentary lifestyle. Future research is required to develop psychotropic drugs that have lower risk of metabolic syndrome and to evaluate the efficacy and safety of pharmacological agents such as metformin and GLP-1 agonists in treating the syndrome in psychiatric patients.

\section{References}

Alberti KG, Eckel RH, Grundy SM, et al (2009) Harmonizing the metabolic syndrome: a joint interim statement of the International Diabetes Federation Task Force on Epidemiology and Prevention; National Heart, Lung, and Blood Institute; American Heart Association; World Heart Federation; International Atherosclerosis Society; and International Association for the Study of Obesity. Circulation, 120: 1640-5.

Anagnostis P, Athyros VG, Tziomalos K, et al (2009) Clinical review: the pathogenetic role of cortisol in the metabolic syndrome: a hypothesis. Journal of Clinical Endocrinology \& Metabolism, 94: 2692-701.

Attvall S, Fowelin J, Lager I, et al (1993) Smoking induces insulin resistance: a potential link with the insulin resistance syndrome. Journal of Internal Medicine, 233: 327-32.

Balkau B, Charles MA (1999) Comment on the provisional report from the WHO consultation. European Group for the Study of Insulin Resistance (EGIR). Diabetic Medicine, 16: 442-3.

Bench CJ, Friston KJ, Brown RG, et al (1992) The anatomy of melancholia: focal abnormalities of cerebral blood flow in major depression. Psychological Medicine, 22: 607-15.

Bjomtorp P, Rosmond R (2000) The metabolic syndrome - a neuroendocrine disorder? British Journal of Nutrition, 83 (suppl 1): S49-57.

obese individuals who have a BMI between 35 comorbidity (Blackburn 2009). It should be noted that surgery reduces the transit time and surface area for absorption of drugs in the gastrointestinal tract, and these postoperative effects on the pharmacokinetics of psychotropic drugs have yet to be investigated in depth.

\section{Conclusions}

The bidirectional interplay between metabolic syndrome and psychiatric disorders, which involves genetic, pharmacological, inflammatory, endocrinological and behavioural factors, is complex and clinically challenging. Screening,
Blackburn GL, Hutter MM, Harvey AM, et al (2009) Expert panel on weight loss surgery: executive report update. Obesity (Silver Spring), 17: 842-62.

Bloomgarden ZT (2004) Definitions of the insulin resistance syndrome. The 1st World Congress on the Insulin Resistance Syndrome. Diabetes Care, 27: 824-30.

Capuron L, Su S, Miller AH, et al (2008) Depressive symptoms and metabolic syndrome: is inflammation the underlying link? Biological Psychiatry, 64: 896-900.

Chang $\mathrm{HH}$, Chou $\mathrm{CH}$, Chen PS, et al (2009) High prevalence of metabolic disturbances in patients with bipolar disorder in Taiwan. Journal of Affective Disorders, 117: 124-9.

Cheng C, Chiu HJ, Loh EW, et al (2012) Association of the ADRA1A gene and the severity of metabolic abnormalities in patients with schizophrenia. Progress in Neuro-Psychopharmacology \& Biological Psychiatry, 36: 205-10.

Chi MH, Chang hH, Tzeng NS, et al (2013) The prevalence of metabolic syndrome in drug-naïve bipolar II disorder patients before and after twelve week pharmacological intervention. Journal of Affective Disorders, 146: 79-83. 
Chokka P, Tancer M, Yeragani VK (2006) Metabolic syndrome: relevance to antidepressant treatment. Journal of Psychiatry Neuroscience, 31: 414.

Christoph U, Correll MD, Anne M, et al (2006) Metabolic syndrome and the risk of coronary heart disease in 367 patients treated with second generation antipsychotic drugs. Journal of Clinical Psychiatry, 67: 575-83.

Connolly M, Kelly C (2005) Lifestyle and physical health in schizophrenia. Advances in Psychiatric Treatment, 11: 125-32.

Correll CU, Frederickson AM, Kane JM, et al (2007) Does antipsychotic polypharmacy increase the risk for metabolic syndrome? Schizophrenia Research, 89: 91-100.

de Hert M, Dekker JM, Wood D, et al (2009) Cardiovascular disease and diabetes in people with severe mental illnesses: position statement from the European Psychiatric Association (EPA), supported by the European Association for the study of Diabetes (EASD) and the European Society of Cardiology (ESC). European Psychiatry, 24: 412-24.

de la Torre JC (2010) The vascular hypothesis of Alzheimer's disease: bench to bedside and beyond. Neurodegenerative Diseases, 7: 116-21.

Expert Panel on Detection, Evaluation, and Treatment of High Blood Cholesterol in Adults (2001) Executive Summary of the Third Report of the National Cholesterol Education Program (NCEP) Expert Panel on Detection, Evaluation, and Treatment of High Blood Cholesterol in Adults (Adult Treatment Panel III). JAMA, 285: 2486-97.

Fagiolini A, Frank E, Scott JA, et al (2005) Metabolic syndrome in bipolar disorder: findings from the Bipolar Disorder Centre for Pennsylvanians. Bipolar Disorder, 7: 424-30

Fernandez-Egea E, Bernardo M, Parellada E, et al (2008a) Glucose abnormalities in the siblings of people with schizophrenia. Schizophrenia Research, 103: 110-3.

Fernandez-Egea E, Miller B, Bernardo M, et al (2008b) Parental history of type 2 disbetes in patients with nonaffective psychosis. Schizophrenia Research, 98: 302-6.

Fernandez-Egea E, Miller B, Garcia-Rizo C, et al (2011) Metabolic effects of olanzapine in patients with newly diagnosed psychosis. Journal of Clinical Psychopharmacology, 31: 154-9.

Fleischhacker WW, Heikkinen ME, Olie JP, et al (2010) Effects of adjunctive treatment with aripiprazole on body weight and clinical efficacy in schizophrenia patients treated with clozapine: a randomized, double-blind, placebo-controlled trial. International Journal of Neuropsychopharmacology, 13: 1115-25.

Gebhardt S, Theisen FM, Haberhausen M, et al (2010) Body weight gain induced by atypical antipsychotics: an extension of the monozygotic twin and sib pair study. Journal of Clinical Pharmacy and Therapeutics, 35: 207-11.

Goeb JL, Marco S, Duhamel A, et al (2010) Metabolic side effects of risperidone in early onset schizophrenia (Abstract) [Article in French]. Encephale, 36: 242-52.

Grundy SM, Brewer Jr HB, Cleeman JL, et al (2004) Definition of metabolic syndrome: report of the National Heart, Lung, and Blood Institute/ American Heart Association conference on scientific issues related to definition. Arteriosclerosis, Thrombosis, and Vascular Biology, 24: e13-8.

Gurnell M (2001) Endocrinology: Medical Masterclass. Royal College of Physicians

Hansen T, Ingason A, Djurovic S, et al (2011) At risk variant in TCF7L2 for type 2 diabetes increases risk of schizophrenia. Biological Psychiatry, 70: 59-63.

Heppner PS, Lohr JB, Kash TP, e al (2012) Metabolic syndrome: relative risk associated with post-traumatic stress disorder (PTSD) severity and antipsychotic medication use. Psychosomatics, 53: 550-8.

Holt R, Abdelrahman T, Hirsch M, et al (2010) The prevalence of undiagnosed metabolic abnormalities in people with serious mental illnesses. Journal of Psychopharmacology, 24: 867-73.

Hope S, Melle I, Aukrust P, et al (2009) Similar immune profile in bipolar disorder and schizophrenia: Selective increase in soluble tumor necrosis factor receptor I and von Willebrand factor. Bipolar Disorder, 11: 726-34.

Howren MB, Lamkin DM, Suls J (2009) Associations of depression with C-reactive protein, IL-1 and IL-6: a meta-analysis. Psychosomatic Medicine, 71: 171-86
International Diabetes Federation (2006) The IDF Consensus Worldwide Definition of the Metabolic Syndrome. IDF.

Kahl KG, Bens S, Ziegler K, et al (2006) Cortisol, the cortisoldehydroepiandrosterone ratio, and pro-inflammatory cytokines in patients with current major depressive disorder comorbid with borderline personality disorder. Biological Psychiatry, 59: 667-71.

Kahl KG, Greggersen W, Schweiger U, et al (2013) Prevalence of the metabolic syndrome in patients with borderline personality disorder: results from a cross-sectional study. European Archives of Psychiatry and Clinical Neuroscience, 263: 205-13.

Kassi E, Pervanidou P, Kaltsas G, et al (2011) Metabolic syndrome: definition and controversies. BMC Medicine, 9: 48

Kim YK, Jung HG, Myint AM, et al (2007) Imbalance between proinflammatory and anti-inflammatory cytokines in bipolar disorder. Journal of Affective Disorders, 104: 91-5.

Kim MK, Kim SH, Yu HS, et al (2012) The effect of clozapine on the AMPK-ACC-CPT1 pathway in the rat frontal cortex. International Journal of Neuropsychopharmacology, 15: 907-17.

Koivistoinen T, Hutri-Kahonen N, Juonala M, et al (2009) Metabolic syndrome and carotid intima media thickness in the Health 2000 Survey. Atherosclerosis, 204: 276-81.

Koponen HJ, Hakko HH, Saari KM, et at (2010) The prevalence and predictive value of individual criteria for metabolic syndrome in schizophrenia: a Northern Finland 1966 Birth Cohort Study. World Journal of Biological Psychiatry, 11: 262-7.

Kral JG, Buckley MC, Kissileff HR, et al (2001) Metabolic correlates of eating behaviour in severe obesity. International journal of obesity and related metabolic disorders, 25: 258-64.

Lee DH, Blomhoff R, Jacobs DR Jr (2004) Is serum gamma glutamyltransferase a marker of oxidative stress? Free Radical Research, 38: $535-9$

Lee NY, Kim SH, Cho B, et al (2010) Patients taking medications for bipolar disorder are more prone to metabolic syndrome than Korea's general population. Progress in Neuropsychopharmacology and Biological Psychiatry, 34: 1243-9.

Lee AK, Bishop JR (2011) Pharmacogenetics of leptin in antipsychoticassociated weight gain and obesity-related complications. Pharmacogenomics, 12: 999-1016.

Maayan L, Vakhrusheva J, Correll C (2010) Effectiveness of medications used to attenuate antipsychotic-related weight gain and metabolic abnormalities: a systematic review and meta-analysis. Neuropsychopharmacology, 35: 1520-30.

McElroy SL, Guerdjikova Al, Martens B, et al (2009) Role of antiepileptic drugs in the management of eating disorders. CNS Drugs, 23: 139-56.

McEvoy JP, Meyer JM, Goff DC, et al (2005) Prevalence of the metabolic syndrome in patients with schizophrenia: baseline results from the Clinical Antipsychotic Trials of Intervention Effectiveness (CATIE) schizophrenia trial and comparison with national estimates from NHANES III. Schizophrenia Research, 80: 9-18.

McLaughlin T, Abbasi F, Lamendola C, et al (2002) Differentiation between obesity and insulin resistance in the association with C-reactive protein. Circulation, 106: 2908-12.

McIntyre RS, Woldeyohannes HO, Soczynska JK, et al (2010) The rate of metabolic syndrome in euthymic Canadian individuals with bipolar I/II disorder. Advances in Therapy, 27: 828-36.

Mclntyre RS, Alsuwaidan M, Goldstein BI, et al (2012) The Canadian Network for Mood and Anxiety Treatments (CANMAT) task force recommendations for the management of patients with mood disorders and comorbid metabolic disorders. Annals of Clinical Psychiatry, 24: 69-81.

Musselman DL, Evans DL, Nemeroff CB (1998) The relationship of depression to cardiovascular disease: epidemiology, biology, and treatment. Archives of General Psychiatry, 55: 580-92.

Na KS, Kim WH, Jung HY, et al (2012) Relationship between inflammation and metabolic syndrome following treatment with paliperidone for schizophrenia. Progress in Neuro-Psychopharmacology \& Biological Psychiatry, 39: 295-300.

\section{MCO answers}

$1 d \quad 2 d \quad 3 c \quad 4$ e 5 a 
Nauck MA (2011) Incretin-based therapies for type 2 diabetes mellitus: properties, functions, and clinical implications. American Journal of Medicine, 124 (1 suppl): S3-18.

Newcomer JW (2007) Metabolic syndrome and mental illness. American Journal of Managed Care, 13 (suppl 7): S170-7.

Oh JH, Kim TH, Sohn YW, et al (2011) Association of serum alanine aminotransferase and $\gamma$-glutamyltransferase levels within the reference range with metabolic syndrome and non-alcoholic fatty liver disease. Korean Journal of Hepatology, 17: 27-36.

Papanastasiou E (2012) Interventions for the metabolic syndrome in schizophrenia: a review. Therapeutic Advances in Endocrinology and Metabolism, 3: 141-62

Park SH, Kim BI, Yun JW, et al (2004) Insulin resistance and C-reactive protein as independent risk factors for non-alcoholic fatty liver disease in non-obese Asian men. Journal of Gastroenterology and Hepatology, 19: $694-8$

Paslakis G, Gilles M, Lederbogen F, et al (2011) The effect of a 4 week treatment with reboxetine on metabolic parameters of depressed inpatients. European Archives of Psychiatry and Neurological Sciences, 261: 173-7.

Patel SB, Reams GP, Spear RM, et al (2008) Leptin: linking obesity, the metabolic syndrome, and cardiovascular disease. Current Hypertension Reports, 10: 131-7.

Potenza MV, Mechanick JI (2009) The metabolic syndrome: definition, global impact, and pathophysiology. Nutrition in Clinical Practice, 24: 560-77.

Praharaj SK, Jana AK, Goyal N, et al (2011) Metformin for olanzapineinduced weight gain: a systematic review and meta-analysis. British Journal of Clinical Pharmacology, 71: 377-82.

Purnell JQ, Kahn SE, Samuels MH, et al (2009) Enhanced cortisol production rates, free cortisol, and 11beta-HSD-1 expression correlate with visceral fat and insulin resistance in men: effect of weight loss. American Journal of Physiology, Endocrinology and Metabolism, 296: E351-7.

Reynolds GP, Templeman LA, Zhang ZJ (2005) The role of 5-HT2C receptor polymorphisms in the pharmacogenetics of antipsychotic drug treatment Progress in Neuro-Psychopharmacology \& Biological Psychiatry, 29: 1021-8

Roberts RO, Geda YE, Knopman DS, et al (2010) Metabolic syndrome, inflammation, and nonamnestic mild cognitive impairment in older persons: a population-based study. Alzheimer Disease and Associated Disorders, 24: 11-8.
Ruzickova M, Slaney C, Garnham J, et al (2003) Clinical features of bipolar disorder with and without comorbid diabetes mellitus. Canadian Journal of Psychiatry, 48: 458-61.

Saddichha S, Manjunatha N, Ameen S, et al (2008) Metabolic syndrome in first episode schizophrenia: a randomized double-blind controlled, short term perspective study. Schizophrenia Research, 101: 266-72.

Skilton MR, Moulin P, Terra JL, et al (2007) Associations between anxiety, depression, and the metabolic syndrome. Biological Psychiatry, 62: $1251-7$.

Sutherland JP, McKinley B, Eckel RH (2004) The metabolic syndrome and inflammation. Metabolic Syndrome and Related Disorders, 2: 82-104.

Takeuchi T, Nakao M, Nomura K, et al (2009) Association of the metabolic syndrome with depression and anxiety in Japanese men: a 1-year cohort study. Diabetes/Metabolism Research and Reviews, 25: 762-7.

Taylor AE, Hubbard J, Anderson EJ (1999) Impact of binge eating on metabolic and leptin dynamics in normal young women. Journal of Clinical Endocrinology \& Metabolism, 84: 428-34.

Une K, Takei YA, Tomita N, et al (2011) Adiponectin in plasma and cerebrospinal fluid in $\mathrm{MCl}$ and Alzheimer's disease. European Journal of Neurology, 18: 1006-9

van Himbergen TM, Beiser AS, Ai M, et al (2012) Biomarkers for insulin resistance and inflammation and the risk for all-cause dementia and Alzheimer disease: results from the Framingham Heart Study. Archives of Neurology, 69: 594-600.

Vuksan-Cusa B, Sagud M, Jakovljevic M, et al (2012) Association between $\mathrm{C}$-reactive protein and homocysteine with the subcomponents of metabolic syndrome in stable patients with bipolar disorder and schizophrenia. Nordic Journal of Psychiatry, PMID: 23228159 [epub ahead of print]

Wojciech J, Szmitkowski M (2007) Effects of ethanol on metabolic syndrome. Polish Archives of Internal Medicine, 117: 306-11.

World Health Organization (1999) Definition, Diagnosis and Classification of Diabetes Mellitus and its Complications. Report of a WHO Consultation. Part 1: Diagnosis and Classification of Diabetes Mellitus. World Health Organization

Yaffe K, Kanaya A, Lindquist K, et al (2004) The metabolic syndrome, inflammation, and risk of cognitive decline. JAMA, 292: 2237-42.

Yates KF, Sweat V, Yau PL, et al (2012) Impact of metabolic syndrome on cognition and brain: a selected review of the literature. Arteriosclerosis, Thrombosis, and Vascular Biology, 32: 2060-7.

\section{MCQs}

Select the single best option for each question stem

\section{Which of the following is least likely to be measured in people with severe mental} illness?

a Blood pressure

b Glucose

c Lipids

d Weight

e None of the above.

2 In bipolar disorder, metabolic syndrome tends to be associated with:

a early age at first treatment for the affective episode

b early onset of first manic episode c less severe first affective episode

d more lifetime affective episodes

e shorter duration of bipolar disorder, especially type II.

3 When a psychiatric patient presents with metabolic syndrome, it is inappropriate to:

a consider adjusting the psychotropic medication according to its cardiometabolic risk profile and psychiatric condition

b engage the patient in support groups to increase their motivation to lose weight

c immediately refer patient to a specialist to manage the metabolic syndrome

$d$ involve family members early in the treatment

e educate the patient about healthy lifestyle habits and diet.
4 The possible mechanisms that metabolic syndrome contributes to dementia include:

a impaired cerebrovascular reactivity

b impaired glucose metabolism

c neuroinflammation

d oxidative stress

e all of the above.

5 Triglyceride and LDL cholesterol levels in patients with schizophrenia and metabolic syndrome can be reduced by a combination of clozapine and:

a aripiprazole

b fluoxetine

c lithium

d methylphenidate

e quetiapine. 